\title{
Assessment of Anatomical Variation of Coronary Arteries for Sudanese Patients Using Cardiac Computed Tomography Angiography
}

\author{
Salem Saeed Alghamdi, $\mathrm{PhD}^{1}$; Rowa Aljondi, $\mathrm{PhD}^{1}$; Ikhlas Abdelaziz, $\mathrm{PhD}^{1}$; \\ Mohamed Yousef, $\mathrm{PhD}^{2}$; Mustafa Z. Mahmoud, $\mathrm{PhD}^{3 *}$; Mugtaba Elgazali, $\mathrm{MSc}^{4}$; \\ Abdulrahman Tajaldeen, $\mathrm{PhD}^{5}$ \\ ${ }^{1}$ University of Jeddah, College of Applied Medical Sciences, Department of Medical Imaging and \\ Radiation Sciences, Jeddah, Saudi Arabia \\ ${ }^{2}$ Radiologic Sciences Program, Batterjee Medical College, Jeddah, Saudi Arabia \\ ${ }^{3}$ Radiology and Medical Imaging Department, College of Applied Medical Sciences, Prince Sattam bin \\ Abdulaziz University, Al-Kharj, Saudi Arabia \\ ${ }^{4}$ College of Medical Radiological Science, Sudan University of Science and Technology, Khartoum, Sudan \\ ${ }^{5}$ Radiological Science Department, College of Applied Medical Science, Imam Abdulrahman Bin Faisal \\ University, Dammam, Saudi Arabia
}

\begin{abstract}
Background: The aims of this study were to assess the prevalence of anatomical variation of coronary arteries among Sudanese patients using cardiac computed tomography angiography (CTA) and to identify the pathological finding for patients undergoing cardiac CTA.

Methods and Results: This prospective cohort study was conducted with 87 Sudanese patients subjected to cardiac CTA examination, using a 64-slice CT scanner (Aquillon 64, Toshiba Medical Systems, Tochigi, Japan) with retrospective ECG gating. Data were collected from 3D reconstruction at optimal phase. The numbers of diagonal and obtuse marginal branches were counted. The presence of ramus intermedius and origination of the right coronary artery (RCA) from right coronary sinus (RCS) were also reported.

Findings showed that most patients had one (33.3\%) or two (47.1\%) diagonal artery branches. In addition, $3.4 \%$ of patients had no diagonal branches, $12.6 \%$ had three and $3.4 \%$ had more than three diagonal branches arising from the left anterior descending artery (LAD). About $8 \%$ of our patients had no obtuse marginal branch. The majority of patients had one, two and three branches of the obtuse marginal arising from a left circumflex artery, which amounted to $24.1 \%, 41.4 \%$ and $25.3 \%$, respectively. Only one patient had more than three obtuse marginal branches. Furthermore, $94.3 \%$ of patients presented with RCA originating from RCS, and $5.7 \%$ of patients had an ectopic origin of the left coronary artery (LCA).

Conclusion: This study concluded that there is a wide variation in the number of diagonal and obtuse marginal branches arising from the left circumflex artery among Sudanese patients. Ramus intermedius was the most common anatomical variation affecting the coronary artery. Ectopic origination of the RCA is a very rare condition in Sudanese patients. (International Journal of Biomedicine. 2020;10(2):101-103.)
\end{abstract}

Key Words: anatomical variation $\bullet$ coronary arteries $\bullet$ cardiac computed tomography angiography

\section{Abbreviations}

CAD, coronary artery disease; CTA, computed tomography angiography; ECG, electrocardiography; LAD, left anterior descending artery; LCA, left coronary artery; RCA, right coronary artery; RCS, right coronary sinus; ROI, region of interest; 3D, three-dimensional 


\section{Introduction}

The arterial supply to the heart is provided by the right and left coronary arteries, which arise from the ascending aorta immediately above the aortic valve. The coronary arteries and their major branches are distributed over the surface of the heart, lying within sub-epicardial connective tissue. The RCA arises from the anterior aortic sinus of the ascending aorta and runs forward between the pulmonary trunk and the right auricle. It descends almost vertically in the right atrioventricular groove, and at the inferior border of the heart, it continues posteriorly along the atrioventricular groove to anastomose with the LCA in the posterior interventricular groove. The branches from the RCA supply the right atrium and right ventricle, parts of the left atrium and left ventricle, and the atrioventricular septum..$^{(1,2)}$ The LCA, which is usually larger than the RCA, supplies the major part of the heart, including the greater part of the left atrium, left ventricle, and ventricular septum. It arises from the left posterior aortic sinus of the ascending aorta and passes forward between the pulmonary trunk and the left auricle. It then enters the atrioventricular groove and divides into an anterior interventricular branch and a circumflex branch. ${ }^{(1,3)}$

Cardiac CTA has emerged as a less invasive imaging modality for the diagnosis of CAD and is often used to avoid conventional coronary angiography, particularly, in low- and intermediate-risk patients. ${ }^{(4-6)}$ Continuous improvements in CT detector technology and in temporal and spatial resolution that have resulted in clinical examinations with cardiac $\mathrm{CT}$ are similar to examinations obtainable with conventional catheter coronary angiography. ${ }^{(7,8)}$

The aims of this study were to assess the prevalence of anatomical variation of coronary arteries among Sudanese patients using cardiac computed tomography angiography (CTA) and to identify the pathological finding for patients undergoing cardiac CTA.

\section{Materials and Methods}

After we received approval from the local ethics committee, a group of 87 Sudanese patients undergoing cardiac CTA, presenting at the Radiology Department, were recruited between March 2019 and April 2020 for this prospective study. The current study adhered to the Declaration of Helsinki and Title 4, US Code of Federal Regulations, Part 46, Protection of Human Subjects.

All cardiac CTA examinations were performed using a 64-slice CT scanner (Aquillon 64, Toshiba Medical Systems, Tochigi, Japan) with retrospective ECG gating. Each patient was injected with $80-85 \mathrm{~mL}$ high iodine concentration contrast media with a flow rate of $5 \mathrm{~mL} / \mathrm{s}$. In addition, an injection of $20 \mathrm{~mL}$ saline solution was given. Scanning timing was determined by the automated bolus-tracking technique by placing the ROI over the proximal descending aorta and setting the trigger threshold to $180 \mathrm{HU}$. Images were reconstructed at the optimal phase and transferred to another workstation. The 3D reconstruction was performed with high resolution and multiple views in order to count the number of diagonal and obtuse marginal branches in a coronary artery. The presence of ramus intermedius and the origin of the RCA from RCS were reported. One experienced consultant radiologist interpreted images for diagnosis, while all images obtained from patients with surgical bypass and stents were excluded.

Statistical analysis was performed using the standard Statistical Package for the Social Sciences (SPSS Inc., Chicago, IL, USA) version 20 for windows.

\section{Results and Discussion}

Table 1 shows the distribution of gender in our study samples. Of the 87 patients, there were $42(48 \%)$ males and $45(51 \%)$ females. The distribution of age groups in our study patients is shown in Table 2. The age group of 40-50 years $(21 \%)$ had cardiac CTA performed with minimum frequency. On the other hand, the age group of more than 60 years $(42.5 \%)$ had a higher percentage due to the increased risk of CAD with the increase of patient age, as reported by Huxley et al. ${ }^{(9)}$

Table 1.

The distribution of gender in study samples

\begin{tabular}{|l|c|c|}
\hline \multicolumn{1}{|c|}{ Gender } & Frequency & Percentage \\
\hline Male & 42 & $48.3 \%$ \\
\hline Female & 45 & $51.7 \%$ \\
\hline Total & 100 & $100 \%$ \\
\hline
\end{tabular}

Table 2.

The distribution of various age groups

\begin{tabular}{|l|c|c|}
\hline \multicolumn{1}{|c|}{ Age groups } & Frequency & Percentage \\
\hline $40-50$ & 19 & $21.8 \%$ \\
\hline $50-59$ & 31 & $35.6 \%$ \\
\hline More than 60 & 37 & $42.5 \%$ \\
\hline Total & 100 & $100 \%$ \\
\hline
\end{tabular}

As shown in Table 3, a total of 3(3.4\%) patients had no diagonal branch, 29(33.3\%) patients presented with one diagonal, 41(47.1\%) patients had two diagonal branches, $11(12.6 \%)$ patients had three diagonal branches, and 3(3.4\%) patients had more than three diagonal branches arising from the LAD. These results are in agreement with a previous study by Abdelrahman et al. ${ }^{(10)}$

Table 4 shows that $7(8 \%)$ patients presented with no obtuse marginal branches, 21(24.1\%) patients had one obtuse marginal branch, 36(41.4\%) patients had two obtuse marginal branches, $22(25.3 \%)$ patients had three obtuse marginal branches, and $1(1.1 \%)$ patient had more than three obtuse marginal branches arising from the left circumflex artery. These results were not similar to a previous study conducted by Abdelrahman et al., ${ }^{(10)}$ in which the researchers found that most of their study samples had one obtuse marginal branch. This difference was due to the large age groups distributed in their study samples. 
Table 3.

Distribution of the diagonal branches

\begin{tabular}{|l|c|c|}
\hline \multicolumn{1}{|c|}{ Diagonal branches } & Frequency & Percentage \\
\hline Absent & 3 & $3.4 \%$ \\
\hline One & 29 & $33.3 \%$ \\
\hline Two & 41 & $47.1 \%$ \\
\hline Three & 11 & $12.6 \%$ \\
\hline More than three & 3 & $3.4 \%$ \\
\hline Total & 87 & $100 \%$ \\
\hline
\end{tabular}

Table 4.

Distribution of obtuse marginal branches

\begin{tabular}{|l|c|c|}
\hline Obtuse marginal branches & Frequency & Percentage \\
\hline Absent & 7 & $8.0 \%$ \\
\hline One & 21 & $24.1 \%$ \\
\hline Two & 36 & $41.4 \%$ \\
\hline Three & 22 & $25.3 \%$ \\
\hline More than three & 1 & $1.1 \%$ \\
\hline Total & 87 & $100 \%$ \\
\hline
\end{tabular}

Table 5 demonstrates that about 17(19.5\%) Sudanese patients in the current study had no ramus intermedius branches arising from the LCA, compared to the rest of the $70(80.5 \%)$ patients, who presented with intermedius branches arising from the LCA. Table 6 shows that $82(94.3 \%)$ patients had the RCA originating from RCS and 5(5.7\%) patients had ectopic origin from the LCA. Both findings presented in Table 5 and Table 6 were in line with other studies conducted by Abdelrahman et al. ${ }^{(10)}$ and Erol et al. ${ }^{(11)}$

Table 5.

The frequency (n) and percentage (\%) of patients with and without ramus intermedius branches arising from the $L C A$

\begin{tabular}{|l|c|c|}
\hline \multicolumn{1}{|c|}{ Ramus intermedius branch } & Frequency & Percentage \\
\hline Patients with ramus intermedius & 70 & $80.5 \%$ \\
\hline Patients without ramus intermedius & 17 & $19.5 \%$ \\
\hline Total & 87 & $100 \%$ \\
\hline
\end{tabular}

Table 6.

RCA origination in the study samples

\begin{tabular}{|l|c|c|}
\hline \multicolumn{1}{|c|}{ RCA origin } & Frequency & Percentage \\
\hline Normal from RCS & 82 & $94.3 \%$ \\
\hline From LCA & 5 & $5.7 \%$ \\
\hline Total & 87 & $100 \%$ \\
\hline
\end{tabular}

\section{Conclusion}

The present study concluded that there is a wide variation in numbers of diagonal branches arising from the left anterior descending and on obtuse marginal branches arising from the left circumflex artery in Sudanese patients. Finally ectopic origination of the RCA is a very rare condition in Sudanese patients.

\section{Competing Interests} interests.

The authors declare that they have no competing

\section{Acknowledgements}

This publication was supported by the Deanship of Scientific Research at Prince Sattam bin Abdulaziz University, Alkharj, Saudi Arabia.

\section{References}

1. Snell RS. Clinical anatomy by regions. 9th ed. Philadelphia: Lippincott Williams \& Wilkins; 2011.

2. Germann WJ, Cindy L. Stanfield CL. Principles of human physiology. Har/Cdr ed. San Francisco: Benjamin Cummings; 2002.

3. Kelley LL, Petersen CM. Sectional anatomy for imaging professionals. 4th edition. Maryland Heights, Missouri: Mosby; 2018.

4. Durst R, Bogot N, Gilon D, Drenger B. Potential role for coronary computerized angiography for assessing preoperative ischemic risk. J Cardiothorac Vasc Anesth. 2010;24(2):34855. doi: 10.1053/j.jvca.2009.12.007.

5. Sun $\mathrm{Z}, \mathrm{Ng} \mathrm{KH}$. Multislice CT angiography in cardiac imaging. Part II: clinical applications in coronary artery disease. Singapore Med J. 2010;51(4):282-89.

6. Sun Z. Cardiac Imaging Modalities in the Diagnosis of Coronary Artery Disease. J Clin Exp Cardiolog. 2013;S6: 1-4. doi: 10.4172/2155-9880.S6-e001

7. Kitagawa K, Lardo AC, Lima JAC, George RT. Prospective ECG-gated 320 row detector computed tomography: implications for CT angiography and perfusion imaging. Int $\mathrm{J}$ Cardiovasc Imaging. 2009;25(2):201-8. doi: 10.1007/s10554009-9433-6.

8. Romans L. Computed Tomography for Technologists: A comprehensive text. 2nd ed. Philadelphia: Lippincott Williams \& Wilkins; 2018.

9. Huxley RR, Barzi F, Lam TH, Czernichow S, Fang $\mathrm{X}$, Welborn $\mathrm{T}$, et al. Isolated low levels of high-density lipoprotein cholesterol are associated with an increased risk of coronary heart disease: an individual participant data meta-analysis of 23 studies in the Asia-Pacific region. Circulation. 2011;124(19):2056-64. doi: 10.1161/ CIRCULATIONAHA.111.028373.

10. Abdelrahman SF, Salem MA, Mostafa NDE, Elbohy AEM. Coronary arteries variants \& congenital anomalies; using MDCT to assess their prevalence in 1000 of the Egyptian population. Egypt J Radiol Nucl Med. 2015;46(4):885-892. doi: 10.1016/j.ejrnm.2015.09.012.

11. Erol C, Koplay M, Paksoy Y. Evaluation of anatomy, variation and anomalies of the coronary arteries with coronary computed tomography angiography. Anatol J Cardiol. 2013;13(2):154-64. Doi: 10.5152/akd.2013.041.

*Corresponding author: Professor Dr. Mustafa Z. Mahmoud, Radiology and Medical Imaging Department, College of Applied Medical Sciences, Prince Sattam bin Abdulaziz University, E-mail. m.alhassen@psau.edu.sa, PO Box: 422, Zip Code: 11942, Al-Kharj, Saudi Arabia. 